\title{
Health Promoting Behaviors and Factors related to Lifestyle among Turkish Workers and Occupational Health Nurses' Responsibilities in their Health Promoting Activities
}

\author{
Ayşe BEŞER*, Zuhal BAHAR and Dilek BÜYÜKKAYA
}

School of Nursing, Dokuz Eylul University, Izmir, Turkey

Received October 11,2005 and accepted July 21, 2006

\begin{abstract}
The purposes of this study were to describe health-promotion lifestyle profile of $\mathbf{2 6 4}$ Turkish workers, to determine the factors which affect their lifestyle and to describe occupational health nurses' responsibilities in their health promoting activities to compare their profile with those published from other studies using Health- Promoting Lifestyle Profile. This is a descriptive study. Study was conducted in a food industry. 530 workers are working in this workplace. Approximately fifty percent of the workers participated in this study.The convenience sample composed of 264 workers. Data were collected using a questionnaire about socio-demographic features developed by the investigators and Health Promoting Lifestyle Profile developed by Walker et al. Subscales with the highest means in this study were interpersonal support and self-actualization. Compared to workers reported from other studies, Turkish workers got low scores of self-actualization, nutrition, interpersonal support and stress management. There was no statistically significant difference between total scores and gender, marital status and education. However, there was a statistically significant difference between age and exercise and nutrition. Moreover, as income increased, so did health promoting behaviors. There was a statistically significant difference between perceived health status and importance placed on health and overall health promoting life style and each health promoting behavior. It is important that occupational health nurses identify health behaviors, perceived health status and cultural aspects likely to affect health behaviors among workers. Thus, they may develop effective tools to protect and promote workers' health.
\end{abstract}

Key words: Health behavior, Health promotion, Workers, Occupational health nurses, Life style

\section{Introduction}

Data about health services at workplaces show that workers are exposed to poor health conditions. According to data from Social Insurance Institution (the institution paying money to people who are ill, unemployed or retired. It is financed by money that the government collects from people who work or from their employers like social insurance in Britain), there were a total of $6,076,705$ workers $(1,261,552$

*To whom correspondence should be addressed. females and 4,815,153 males) in Turkey by 2003 . There were 76,668 industrial accidents and 440 workers had occupational diseases. There were 695,779 workers with a mean age of $33 \mathrm{yr}$ who caught a disease which caused disability $^{1)}$. The above mentioned data reveal the priority to protect and promote workers' health. Occupational health nurses should determine health behaviors of workers and develop programs to improve them. There have been limited studies on health promoting behaviors such as health responsibility, self-actualization, health control, stress management, nutrition and exercise and few health promoting 
programs have been developed in Turkey.

Pender developed a health promotion model consisting of three major components: (a) cognitive/perceptual or psychological elements that determine participation in healthpromoting behaviors; (b) modifying circumstances that influence the cognitive-perceptual factors and thus indirectly influence health promoting behaviors; and (c) the likelihood of actions directed toward enhancing or maintaining wellbeing. The cognitive-perceptual factors include importance of health, definition of health; perception of health, selfefficacy, and health status; and benefits of and barriers to health promoting behaviors. Modifying factors are demographic and biologic characteristics, interpersonal influences, and situational and behavioral factors ${ }^{2}$. The health promotion model is intended to explain the occurrence of behaviors directed toward optimizing well-being ${ }^{3)}$.

According to Pender, healthy life style behaviors are selfactualization, health responsibility, exercise, nutrition, interpersonal support and stress management $t^{4}$. Studies on health promoting behaviors have focused on certain groups. For example, Al-Ma' aitah $^{5)}$ investigated health promoting behaviors of Jordanian Muslim women and found that the women got high scores of self-actualization, interpersonal support and nutrition. Ahijevych ${ }^{6}$ reported that AfricanAmerican women received low scores of self-actualization, exercise, nutrition and interpersonal support. Esin noted that Turkish women got high scores of self-actualization and health responsibility and that they got the lowest scores on exercise ${ }^{7}$.

Several studies found some socio-demographic variables related to the practice of health behaviors ${ }^{5-8}$. Duffy ${ }^{9)}$ found that age and education affected healthy life style behaviors. Kuster ${ }^{10)}$ reported that age, education, income, length of residence in the United States and perceived health status correlated significantly and positively with the Spanish HealthPromoting Lifestyle Profile (HPLP) total and subscale scores.

Studies on health promoting behaviors have mostly included people from certain age groups, genders and ethnic origins. However, there have been few studies on employees. Those few studies have enrolled blue collar workers. Dufy ${ }^{9)}$ and Weitzel ${ }^{11)}$ noted that workers got the highest scores from subscales of self-actualization and interpersonal support. Pender ${ }^{2}$ found that workers received the highest scores on self-actualization and exercise, but that they got low scores on health responsibility and stress management. Lusk ${ }^{12)}$ reported that blue collar workers did not have a desirable level of healthy life style behaviors. Kerr ${ }^{13)}$ noted that English speaking Mexican- American workers had lower scores of self-actualization and stress management than the Spanish speaking ones.

$\operatorname{Esin}^{14)}$ in her study on 450 workers established that workers had a moderate level of healthy life style behaviors and that males had high scores of healthy life style behaviors. Moreover, as age, education, work experience and status and income increased so did health promoting behaviors. Tokgö ${ }^{15)}$ in her study on 282 female lecturers discovered that self-actualization and interpersonal support scores were the highest, but health responsibility and exercise scores were the lowest.

Pender $^{2)}$ and Duffy ${ }^{9}$ found age to be positively related to overall health- promoting lifestyle scores. However, Weitzel ${ }^{11)}$ found age to be negatively related to exercise and positively related to health responsibility and nutrition. Lusk $^{12)}$ reported that younger workers had significantly higher scores than the other groups on self actualization, exercise and interpersonal support while older workers had significantly higher scores than the other age groups on health.

Gender has been shown to predict health protective behaviors ${ }^{11,12)}$. In fact, Bagwell ${ }^{16)}$ found out that females had higher scores of health responsibility and interpersonal support than males but that there was no significant difference between age and health promoting behaviors. Pender ${ }^{2}$ found health promoting lifestyle scores were positively associated with female gender. Bagwell ${ }^{16)}$ claimed that there was a significant difference in health responsibility and interpersonal support between men and women, with women having a higher mean score on both behaviors.

Weitzell and Lusk ${ }^{11,12)}$ reported that educational groups were significantly different from the others with respect to mean scores on the HPLP and its subscales, with higher education consistently predicting higher scores. Walker ${ }^{17)}$ included marital status in their analyses, finding marriage to be positively related to nutrition. However, Lusk ${ }^{12)}$ found no relation between marital status and overall scores of HPLP and subscales, which is consistent with the results of a study by Tokgöz ${ }^{15)}$.

Weitzel ${ }^{11)}$ found income to be positively related to health promoting lifestyle and self actualization scores for Hispanic but not a predictor for African Americans or whites.

Pender $^{2}$ found no significant relationship between income and health promoting lifestyle scores. However, Esin ${ }^{14)}$ established that as income increased so did HPLP scores.

Bagwell ${ }^{16)}$ established a significant relation between perception of health and healthy life style behaviors. Angela ${ }^{18)}$ in his integrative review on health promotion noted that ten studies revealed a positive correlation between importance of health and health promoting behaviors. Angela ${ }^{18}$ in his study on 120 health staff reported a positive relation between 
perception of health and healthy life style behaviors, which is compatible with results of the studies by Weitzel, Pender and Walker', 11, 17).

Nursing is part of the health care delivery system and more than in any other discipline a nursing practitioner is in continuous contact with workers and their families. Therefore, occupational health nurses as well as other members of interdisciplinary health teams are more likely to contribute to a positive change in workers' behavior.

The purposes of this study were to describe healthpromotion lifestyle profile of 264 Turkish workers, to determine the factors which affect their lifestyle and to describe occupational health nurses' responsibilities in their health promoting activities to compare their profile with those published from other studies using Health- Promoting Lifestyle Profile.

Exploration of the health promotion practices of Turkish workers will help occupational health nurses when educating and counseling these individuals related to health-promoting activities.

\section{Material and Methods}

Sample

This is a descriptive study. Study was conducted in a food industry. Five hundred thirty workers are working in this workplace. Approximately fifty percent of the workers participated in this study. The convenience sample composed of 264 workers.

The workers were stratified according to the departments where they worked and their education level so that they represent the population. The mean age of the workers was $30.49 \pm 0.41 \mathrm{yr}$. Of all workers, $70 \%$ were male, $30 \%$ females, $56.5 \%$ married and $43.5 \%$ single. Of all males, $18.4 \%$ were primary school graduates, $17.8 \%$ junior school graduates, $41.1 \%$ high school graduates and $22.7 \%$ university graduates. Of all females, $13.9 \%$ were primary school graduates, $11.4 \%$ junior school graduates, $30.4 \%$ high school graduates and $44.3 \%$ university graduates. Out of all workers, $30.8 \%$ had a work experience of $1-5 \mathrm{yr}, 30.0 \% 6-10 \mathrm{yr}$, and $22.1 \%$ ten years more, $13.4 \%$ 0-5 months and 3.7\% 6-11 months. $62.7 \%$ of workers had a moderate income.

\section{Instrument}

The participants were asked their answers of questions about the demographic factors such as age, gender, education, marital status, income and total work experience, all of which were the variables determined by the investigators in view of the literature. Health Promoting Lifestyle Profile Scale was used to collect data about health behaviors. The scale was developed by Walker et al. ${ }^{19)}$. It is composed of 48 items and 6 subscales and consists of questions about health promoting behaviors. The subscales were self-actualization, health responsibility, exercise, nutrition, interpersonal support and stress management. The total score reflects the healthy life-style behavior. Four more items were added to the scale and now the scale is composed of 52 items ${ }^{20)}$. The scale composed of 52 items was used in this study.

Each respondent was asked to rate each item on Likert's 1-to-4 response scale: where: 1 corresponds to never, 2 sometimes, 3 often and 4 regularly. Alpha coefficient reliability of the scale was 0.92 and alpha coefficient reliability of the subscales varied from 0.70 to 0.90 .

The reliability of the scale for Turkish population was tested by $\operatorname{Esin}^{14)}$ and Akça ${ }^{21}$. Alpha coefficient reliability of the scale was 0.91 in Esin's study and 0.90 in Akça's study.

Using a 3-point response format ranging from poor to excellent, participants scored their perceived health status when answering the question "how do you evaluate your health compared to people of the same age as you?".

Using a 5-point response format ranging from none to very much, participants reported what they thought about importance of health when answering the question "to what degree do you give importance to your health?". Data were analyzed using SPSS package program, t test, one way ANOVA test, Tukey analyses.

\section{Procedure}

Data were collected by the interns who received practical training on occupational health nursing. Before data collection, the students were given information about interview techniques and comprehensibility of questions. The questionnaires were distributed to and received back from employees working in the offices during the daytime, but the workers in the production line were interviewed during breaks and lunches. Interviewers explained the purposes and procedures of the study to the participants. The participants were informed that they had the right to refuse participation at any time. They were also informed that the obtained information would be confidential.

Approval was obtained from the ethical committee of the nursing school and the director of Human Resources.

\section{Results}

The mean total HPLP score was $2.4 \pm 0.41$ (Table 1). The participants received the highest mean scores on interpersonal support $(2.7 \pm 0.47)$ and self-actualization (2.7 
Table 1. Comparison of mean HPLP scores from various studies

\begin{tabular}{|c|c|c|c|c|c|c|c|c|}
\hline & $\begin{array}{c}\text { Self- } \\
\text { actualization }\end{array}$ & $\begin{array}{l}\text { Health } \\
\text { responsibility }\end{array}$ & Exercise & Nutrition & $\begin{array}{c}\text { Interpersonal } \\
\text { Support }\end{array}$ & $\begin{array}{c}\text { Stress } \\
\text { Management }\end{array}$ & TOTAL & $\mathrm{N}$ \\
\hline $\begin{array}{l}\text { Mexican-American } \\
\text { female workers } \\
\text { Duffy et al. (1996) }\end{array}$ & $\begin{array}{l}3.1 \\
(0.55)\end{array}$ & $\begin{array}{l}2.3 \\
(0.46)\end{array}$ & $\begin{array}{l}1.9 \\
(0.79)\end{array}$ & $\begin{array}{l}2.5 \\
(0.64)\end{array}$ & $\begin{array}{l}3 \\
(0.57)\end{array}$ & $\begin{array}{l}2.5 \\
(0.58)\end{array}$ & $\begin{array}{l}2.7 \\
(0.46)\end{array}$ & 397 \\
\hline $\begin{array}{l}\text { Predicting Health } \\
\text { Promoting Lifestyles } \\
\text { in the workplace } \\
\text { Pender } \text { et al. (1990) }\end{array}$ & $\begin{array}{c}3.24 \\
(0.45)\end{array}$ & $\begin{array}{c}2.23 \\
(0.54)\end{array}$ & $\begin{array}{c}3.24 \\
(0.59)\end{array}$ & $\begin{array}{l}2.66 \\
(0.67)\end{array}$ & $\begin{array}{c}3.14 \\
(0.55)\end{array}$ & $\begin{array}{c}2.42 \\
(0.52)\end{array}$ & $\begin{array}{c}2.82 \\
(0.39)\end{array}$ & 250 \\
\hline $\begin{array}{l}\text { Blue Collar, Skilled } \\
\text { Trade White Collar } \\
\text { Workers } \\
\text { Lusk et al. (1995) }\end{array}$ & $\begin{array}{c}3.04 \\
(0.49)\end{array}$ & $\begin{array}{c}2.24 \\
(0.57)\end{array}$ & $\begin{array}{c}2.18 \\
(0.79)\end{array}$ & $\begin{array}{c}2.47 \\
(0.63)\end{array}$ & $\begin{array}{c}2.86 \\
(0.57)\end{array}$ & $\begin{array}{c}2.49 \\
(0.54)\end{array}$ & $\begin{array}{c}2.60 \\
(0.47)\end{array}$ & 638 \\
\hline $\begin{array}{l}\text { Health Behaviors of } \\
\text { Lecturers } \\
\text { Tokgöz (2002) }\end{array}$ & $\begin{array}{c}2.81 \\
(0.78)\end{array}$ & $\begin{array}{c}2.39 \\
(0.80)\end{array}$ & $\begin{array}{c}1.96 \\
(0.83)\end{array}$ & $\begin{array}{c}2.52 \\
(0.99)\end{array}$ & $\begin{array}{c}2.95 \\
(0.74)\end{array}$ & $\begin{array}{c}2.48 \\
(0.84)\end{array}$ & $\begin{array}{c}2.53 \\
(0.83)\end{array}$ & 282 \\
\hline $\begin{array}{l}\text { This Study } \\
\text { Beser } \text { et al. }\end{array}$ & $\begin{array}{c}2.74 \\
(0.51)\end{array}$ & $\begin{array}{c}2.30 \\
(0.54)\end{array}$ & $\begin{array}{c}2.02 \\
(0.64)\end{array}$ & $\begin{array}{c}2.40 \\
(0.46)\end{array}$ & $\begin{array}{c}2.78 \\
(0.47)\end{array}$ & $\begin{array}{c}2.39 \\
(0.53)\end{array}$ & $\begin{array}{c}2.45 \\
(0.41)\end{array}$ & 264 \\
\hline
\end{tabular}

\pm 0.51 ) (Table 1). However, the participants got the lowest mean scores on exercise $(2.0 \pm 0.64)$ and health responsibility $(2.3 \pm 0.54)$ and moderate mean scores on nutrition $(2.4 \pm$ $0.46)$ and stress management $(2.3 \pm 0.53)$ (Table 1$)$.

The mean HPLP scores obtained in this study are compared with those from other studies in Table 1 . In the study by Pender et al, received the highest mean total HPLP and selfactualization. However, Turkish workers got low scores on self-actualization. The highest mean scores on health responsibility came from the study on lecturers by Tokgöz and the lowest scores on this subscale came from the study by Pender et al. The mean exercise scores were comparable in all studies except for those reported by Pender et al. The highest mean interpersonal support scores were reported by Pender et al and the lowest mean interpersonal support scores were reported by Lusk et al.

There was no statistically significant difference between total HPLP scores and age. But there was a difference between age and exercise $(p<0.05)$ and nutrition scores $(p<0.05)$ (Table 2).

There was no statistically significant difference between total HPLP and subscale scores and gender, education level and marital status. But the difference between income and total HPLP $(p<0.001)$ and subscale scores was statistically significant (Table 2). As income increased so did positive health behaviors.

There was a statistically significant difference between total work experience and total HPLP scores $(p<0.05)$ and exercise $(p<0.05)$ (Table 3$)$. Further analysis showed that the statistically significant difference resulted from the groups with a total work experience of 6-10 yr.

There was no statistically significant difference in total HPLP scores between blue and white collar workers $(p>0.05)$ (Table 3 ). But there was a statistically significant difference in stress scores $(p<0.05)$. Further analysis showed that the difference resulted from the white collar workers. The white collar workers had lower stress scores than blue collar workers.

There was a statistically significant difference between perceived health status and total HPLP $(p<0.001)$ and subscale scores (Table 4). The participants who perceived their health to be excellent received high scores of HPLP and subscales.

The difference between importance of health and total HPLP $(p<0.001)$ and subscale scores was statistically significant (Table 4). The participants who gave an utmost importance to their health and thought to control their health in the future received higher scores of HPLP and subscales.

\section{Discussion}

We found that Turkish workers did not have many healthy life style behaviors compared to those reported from other studies $^{2,9,12,15)}$. The results may have been influenced by Turkish culture and social structure or characteristics of the study sample. In fact, Duff and Pender included individuals better equipped with knowledge about health promoting 
Table 2. Between socio-demographic features of workers and their HPLP scores

\begin{tabular}{|c|c|c|c|c|c|c|c|c|}
\hline & & $\begin{array}{l}\text { Self- } \\
\text { actualization }\end{array}$ & $\begin{array}{c}\text { Health } \\
\text { Responsibility }\end{array}$ & Exercise & Nutrition & $\begin{array}{c}\text { Interpersonal } \\
\text { Support }\end{array}$ & $\begin{array}{c}\text { Stress } \\
\text { Management }\end{array}$ & Total \\
\hline \multicolumn{9}{|l|}{ Age } \\
\hline $15-19$ & & $\begin{array}{c}2.77 \\
(0.51)\end{array}$ & $\begin{array}{c}2.34 \\
(0.56)\end{array}$ & $\begin{array}{c}1.98 \\
(0.66)\end{array}$ & $\begin{array}{c}2.40 \\
(0.41)\end{array}$ & $\begin{array}{c}2.80 \\
(0.41)\end{array}$ & $\begin{array}{c}2.34 \\
(0.43)\end{array}$ & $\begin{array}{c}2.45 \\
(0.31)\end{array}$ \\
\hline $20-24$ & & $\begin{array}{c}2.79 \\
(0.53)\end{array}$ & $\begin{array}{c}2.30 \\
(0.51)\end{array}$ & $\begin{array}{c}2.14 \\
(0.63)\end{array}$ & $\begin{array}{c}2.40 \\
(0.47)\end{array}$ & $\begin{array}{c}2.77 \\
(0.48)\end{array}$ & $\begin{array}{c}2.43 \\
(0.53)\end{array}$ & $\begin{array}{c}2.48 \\
(0.42)\end{array}$ \\
\hline $25-29$ & & $\begin{array}{c}2.66 \\
(0.47)\end{array}$ & $\begin{array}{c}2.20 \\
(0.51)\end{array}$ & $\begin{array}{c}1.86 \\
(0.58)\end{array}$ & $\begin{array}{c}2.33 \\
(0.46)\end{array}$ & $\begin{array}{c}2.76 \\
(0.49)\end{array}$ & $\begin{array}{c}2.30 \\
(0.55)\end{array}$ & $\begin{array}{c}2.36 \\
(0.41)\end{array}$ \\
\hline $30-34$ & & $\begin{array}{c}2.73 \\
(0.50)\end{array}$ & $\begin{array}{c}2.36 \\
(0.58)\end{array}$ & $\begin{array}{c}2.05 \\
(0.69)\end{array}$ & $\begin{array}{c}2.44 \\
(0.43)\end{array}$ & $\begin{array}{c}2.81 \\
(0.43)\end{array}$ & $\begin{array}{c}2.47 \\
(0.52)\end{array}$ & $\begin{array}{c}2.49 \\
(0.43)\end{array}$ \\
\hline $35-39$ & & $\begin{array}{c}2.84 \\
(0.67)\end{array}$ & $\begin{array}{c}2.41 \\
(0.52)\end{array}$ & $\begin{array}{c}2.11 \\
(0.57)\end{array}$ & $\begin{array}{c}2.62 \\
(0.52)\end{array}$ & $\begin{array}{c}2.79 \\
(0.55)\end{array}$ & $\begin{array}{c}2.45 \\
(0.57)\end{array}$ & $\begin{array}{c}2.55 \\
(0.42)\end{array}$ \\
\hline \multirow[t]{3}{*}{$40-$} & & $\begin{array}{c}3.11 \\
(0.62)\end{array}$ & $\begin{array}{c}2.94 \\
(1.17)\end{array}$ & $\begin{array}{c}3.12 \\
(1.06)\end{array}$ & $\begin{array}{c}3.16 \\
(0.86)\end{array}$ & $\begin{array}{c}3.27 \\
(0.39)\end{array}$ & $\begin{array}{c}3.31 \\
(0.97)\end{array}$ & $\begin{array}{c}3.15 \\
(0.84)\end{array}$ \\
\hline & $\mathrm{F}$ & 0.841 & 1.430 & 2.983 & 2.262 & 0.517 & 2.047 & 2.204 \\
\hline & $p$ & 0.522 & 0.214 & $0.012 *$ & $0.049 *$ & 0.763 & 0.073 & 0.054 \\
\hline \multicolumn{9}{|l|}{ Gender } \\
\hline Male & & $\begin{array}{c}2.74 \\
(0.52)\end{array}$ & $\begin{array}{c}2.29 \\
(0.53)\end{array}$ & $\begin{array}{c}2.04 \\
(0.66)\end{array}$ & $\begin{array}{c}2.40 \\
(0.46)\end{array}$ & $\begin{array}{c}2.77 \\
(80.48)\end{array}$ & $\begin{array}{c}2.40 \\
(0.53)\end{array}$ & $\begin{array}{c}2.45 \\
(0.41)\end{array}$ \\
\hline \multirow[t]{3}{*}{ Woman } & & $\begin{array}{c}2.75 \\
(0.49)\end{array}$ & $\begin{array}{c}2.31 \\
(0.56)\end{array}$ & $\begin{array}{c}1.97 \\
(0.58)\end{array}$ & $\begin{array}{c}2.41 \\
(0.46)\end{array}$ & $\begin{array}{c}2.82 \\
(0.45)\end{array}$ & $\begin{array}{c}2.37 \\
(0.53)\end{array}$ & $\begin{array}{c}2.45 \\
(0.41)\end{array}$ \\
\hline & $\mathrm{t}$ & -0.039 & -0.320 & 0.854 & -0.151 & -0.724 & 0.321 & 0.013 \\
\hline & $p$ & 0.969 & 0.750 & 0.394 & 0.880 & 0.470 & 0.749 & 0.989 \\
\hline \multicolumn{9}{|l|}{ Education } \\
\hline \multicolumn{2}{|l|}{ Primary school } & $\begin{array}{c}2.73 \\
(0.58)\end{array}$ & $\begin{array}{c}2.34 \\
(0.57)\end{array}$ & $\begin{array}{c}1.93 \\
(0.64)\end{array}$ & $\begin{array}{c}2.43 \\
(0.52)\end{array}$ & $\begin{array}{c}2.72 \\
(0.50)\end{array}$ & $\begin{array}{c}2.53 \\
(0.49)\end{array}$ & $\begin{array}{c}2.46 \\
(0.43)\end{array}$ \\
\hline $\begin{array}{l}\text { Junior high } \\
\text { school }\end{array}$ & & $\begin{array}{c}2.78 \\
(0.61)\end{array}$ & $\begin{array}{c}2.35 \\
(0.53)\end{array}$ & $\begin{array}{c}1.98 \\
(0.74)\end{array}$ & $\begin{array}{c}2.40 \\
(0.42)\end{array}$ & $\begin{array}{c}2.75 \\
(0.56)\end{array}$ & $\begin{array}{c}2.42 \\
(0.62)\end{array}$ & $\begin{array}{c}2.46 \\
(0.43)\end{array}$ \\
\hline \multicolumn{2}{|l|}{ High school } & $\begin{array}{c}2.75 \\
(0.49)\end{array}$ & $\begin{array}{c}2.27 \\
(0.53)\end{array}$ & $\begin{array}{c}2.02 \\
(0.60)\end{array}$ & $\begin{array}{c}2.36 \\
(0.46)\end{array}$ & $\begin{array}{c}2.77 \\
(0.44)\end{array}$ & $\begin{array}{c}2.33 \\
(0.55)\end{array}$ & $\begin{array}{c}2.43 \\
(0.40)\end{array}$ \\
\hline \multirow[t]{3}{*}{ University } & & $\begin{array}{c}2.73 \\
(0.45)\end{array}$ & $\begin{array}{c}2.27 \\
(0.54)\end{array}$ & $\begin{array}{c}2.10 \\
(0.64)\end{array}$ & $\begin{array}{c}2.44 \\
(0.46)\end{array}$ & $\begin{array}{c}2.85 \\
(0.43)\end{array}$ & $\begin{array}{c}2.37 \\
(0.48)\end{array}$ & $\begin{array}{c}2.47 \\
(0.41)\end{array}$ \\
\hline & $\mathrm{F}$ & 0.087 & 0.345 & 0.735 & 0.507 & 0.803 & 1.511 & 0.152 \\
\hline & $p$ & 0.967 & 0.793 & 0.532 & 0.678 & 0.493 & 0.212 & 0.928 \\
\hline \multicolumn{9}{|l|}{ Marital Status } \\
\hline Married & & $\begin{array}{c}2.73 \\
(0.54)\end{array}$ & $\begin{array}{c}2.34 \\
(0.57)\end{array}$ & $\begin{array}{c}1.97 \\
(0.66)\end{array}$ & $\begin{array}{c}2.40 \\
(0.47)\end{array}$ & $\begin{array}{c}2.77 \\
(0.50)\end{array}$ & $\begin{array}{c}2.37 \\
(0.56)\end{array}$ & $\begin{array}{c}2.44 \\
(0.44)\end{array}$ \\
\hline \multirow[t]{3}{*}{ Unmarried } & & $\begin{array}{c}2.77 \\
(0.47)\end{array}$ & $\begin{array}{c}2.24 \\
(0.49)\end{array}$ & $\begin{array}{c}2.09 \\
(0.61)\end{array}$ & $\begin{array}{c}2.40 \\
(0.46)\end{array}$ & $\begin{array}{c}2.80 \\
(0.43)\end{array}$ & $\begin{array}{c}2.41 \\
(0.49)\end{array}$ & $\begin{array}{c}2.46 \\
(0.37)\end{array}$ \\
\hline & $\mathrm{t}$ & -0.601 & 1.389 & -1.560 & 0.016 & -0.665 & -0.668 & -0.446 \\
\hline & $p$ & 0.548 & 0.166 & 0.120 & 0.987 & 0.507 & 0.505 & 0.656 \\
\hline \multicolumn{9}{|l|}{ Income } \\
\hline \multicolumn{2}{|l|}{$\begin{array}{l}\text { Income little } \\
\text { of expense }\end{array}$} & $\begin{array}{c}2.59 \\
(0.52)\end{array}$ & $\begin{array}{c}2.22 \\
(0.49)\end{array}$ & $\begin{array}{c}1.84 \\
(0.57)\end{array}$ & $\begin{array}{c}2.29 \\
(0.41)\end{array}$ & $\begin{array}{c}2.62 \\
(0.44)\end{array}$ & $\begin{array}{c}2.20 \\
(0.46)\end{array}$ & $\begin{array}{c}2.30 \\
(0.35)\end{array}$ \\
\hline \multicolumn{2}{|l|}{$\begin{array}{l}\text { Income equal } \\
\text { to expense }\end{array}$} & $\begin{array}{c}2.76 \\
(0.51)\end{array}$ & $\begin{array}{c}2.28 \\
(0.54)\end{array}$ & $\begin{array}{c}2.03 \\
(0.64)\end{array}$ & $\begin{array}{c}2.40 \\
(0.46)\end{array}$ & $\begin{array}{c}2.80 \\
(0.47)\end{array}$ & $\begin{array}{c}2.41 \\
(0.53)\end{array}$ & $\begin{array}{c}2.45 \\
(0.41)\end{array}$ \\
\hline \multirow[t]{3}{*}{$\begin{array}{c}\text { Income excess } \\
\text { of expense }\end{array}$} & & $\begin{array}{c}2.95 \\
(0.43)\end{array}$ & $\begin{array}{c}2.50 \\
(0.59)\end{array}$ & $\begin{array}{c}2.27 \\
(0.68)\end{array}$ & $\begin{array}{c}2.58 \\
(0.50)\end{array}$ & $\begin{array}{c}2.97 \\
(0.44)\end{array}$ & $\begin{array}{c}2.61 \\
(0.57)\end{array}$ & $\begin{array}{c}2.66 \\
(0.42)\end{array}$ \\
\hline & $\mathrm{F}$ & 5.606 & 3.358 & 5.325 & 4.595 & 6.829 & 7.628 & 8.607 \\
\hline & $p$ & $0.004 * *$ & $0.036^{*}$ & $0.005^{* *}$ & $0.011^{*}$ & $0.001 * *$ & $0.001 * *$ & $0.000 * * *$ \\
\hline
\end{tabular}

${ }^{*} p<0.05 ; * * p<0.01 ; * * * p<0.001$ 
Table 3. Features of workers and their HPLP scores

\begin{tabular}{|c|c|c|c|c|c|c|c|}
\hline & $\begin{array}{c}\text { Self- } \\
\text { actualization }\end{array}$ & $\begin{array}{c}\text { Health } \\
\text { Responsibility }\end{array}$ & Exercise & Nutrition & $\begin{array}{c}\text { Interpersonal } \\
\text { Support }\end{array}$ & $\begin{array}{c}\text { Stress } \\
\text { Management }\end{array}$ & Total \\
\hline \multicolumn{8}{|l|}{$\begin{array}{l}\text { Total work } \\
\text { experience }\end{array}$} \\
\hline $0-5$ month & $\begin{array}{c}2.83 \\
(0.58)\end{array}$ & $\begin{array}{c}2.37 \\
(0.54)\end{array}$ & $\begin{array}{c}2.16 \\
(0.75)\end{array}$ & $\begin{array}{c}2.46 \\
(0.48)\end{array}$ & $\begin{array}{c}2.79 \\
(0.46)\end{array}$ & $\begin{array}{c}2.56 \\
(0.52)\end{array}$ & $\begin{array}{c}2.53 \\
(0.40)\end{array}$ \\
\hline 6-11 month & $\begin{array}{c}2.81 \\
(0.72)\end{array}$ & $\begin{array}{c}2.53 \\
(0.57)\end{array}$ & $\begin{array}{c}2.12 \\
(0.56)\end{array}$ & $\begin{array}{c}2.60 \\
(0.59)\end{array}$ & $\begin{array}{c}2.95 \\
(0.52)\end{array}$ & $\begin{array}{c}2.52 \\
(0.27)\end{array}$ & $\begin{array}{r}2.60 \\
(0.41)\end{array}$ \\
\hline $1-5 \mathrm{yr}$ & $\begin{array}{c}2.84 \\
(0.45)\end{array}$ & $\begin{array}{c}2.34 \\
(0.55)\end{array}$ & $\begin{array}{c}2.14 \\
(0.62)\end{array}$ & $\begin{array}{c}2.43 \\
(0.47)\end{array}$ & $\begin{array}{c}2.87 \\
(0.50)\end{array}$ & $\begin{array}{c}2.42 \\
(0.50)\end{array}$ & $\begin{array}{c}2.51 \\
(0.40)\end{array}$ \\
\hline $6-10 \mathrm{yr}$ & $\begin{array}{c}2.62 \\
(0.51)\end{array}$ & $\begin{array}{c}2.22 \\
(0.48)\end{array}$ & $\begin{array}{c}1.84 \\
(0.55)\end{array}$ & $\begin{array}{c}2.33 \\
(0.42)\end{array}$ & $\begin{array}{c}2.71 \\
(0.47)\end{array}$ & $\begin{array}{c}2.26 \\
(0.54)\end{array}$ & $\begin{array}{c}2.34 \\
(0.41)\end{array}$ \\
\hline $11 \mathrm{yr}$ and upper & $\begin{array}{c}2.73 \\
(0.52)\end{array}$ & $\begin{array}{c}2.30 \\
(0.58)\end{array}$ & $\begin{array}{c}1.98 \\
(0.69)\end{array}$ & $\begin{array}{c}2.40 \\
(0.44)\end{array}$ & $\begin{array}{c}2.73 \\
(0.42)\end{array}$ & $\begin{array}{c}2.39 \\
(0.61)\end{array}$ & $\begin{array}{c}2.43 \\
(0.43)\end{array}$ \\
\hline $\mathrm{F}$ & 2.082 & 1.003 & 2.737 & 1.090 & 1.471 & 2.135 & 2.463 \\
\hline$p$ & 0.084 & 0.406 & $0.029^{*}$ & 0.362 & 0.212 & 0.077 & $0.046^{*}$ \\
\hline \multicolumn{8}{|l|}{ Workers } \\
\hline White collar & $\begin{array}{c}2.65 \\
(0.44)\end{array}$ & $\begin{array}{c}2.22 \\
(0.52)\end{array}$ & $\begin{array}{c}1.98 \\
(0.63)\end{array}$ & $\begin{array}{c}2.36 \\
(0.47)\end{array}$ & $\begin{array}{c}2.79 \\
(0.44)\end{array}$ & $\begin{array}{c}2.28 \\
(0.51)\end{array}$ & $\begin{array}{c}2.39 \\
(0.41)\end{array}$ \\
\hline Blue collar & $\begin{array}{c}2.79 \\
(0.54)\end{array}$ & $\begin{array}{c}2.33 \\
(0.54)\end{array}$ & $\begin{array}{c}2.04 \\
(0.65)\end{array}$ & $\begin{array}{c}2.42 \\
(0.46)\end{array}$ & $\begin{array}{c}2.78 \\
(0.49)\end{array}$ & $\begin{array}{c}2.44 \\
(0.54)\end{array}$ & $\begin{array}{c}2.47 \\
(0.41)\end{array}$ \\
\hline $\mathrm{t}$ & -1.961 & -1.472 & -0.632 & -0.943 & 0.085 & -2.329 & -1.527 \\
\hline$p$ & 0.051 & 0.142 & 0.528 & 0.347 & 0.932 & $0.021^{*}$ & 0.128 \\
\hline
\end{tabular}

$* p<0.05$

behaviors into their samples.

Self-actualization determines goals of individuals, their ability to improve their health and to what extent individuals know and satisfy themselves ${ }^{5,15}$. In the present study, workers got the second highest scores on self-actualization, compatible with the results from the studies by Tokgö $\mathrm{z}^{15)}$. Weitzel, Duffy, Pender, Lusk ${ }^{2,9,11,12)}$ also reported that employees received high scores on self-actualization (Table 1). Having a job and a regular income may influence both health behaviors and self-respect. Therefore, it is natural that employees receive high scores on self-actualization.

The extent to which an individual contributes to his/her own health represents his/her health responsibility ${ }^{15)}$. In this study, the participants received low scores on health responsibility, compatible with the results reported from other studies $^{2,9,12,15)}$ (Table 1).

The reason why the workers did not have the desirable degree of health responsibility may be that workers do not consider health controls as a necessity to lead a healthy life. Naturally, if an individual do not perceive his/her health problems, he/she does not make an effort to promote his/ her health ${ }^{22}$. In Turkey, if individuals can do their daily routines and if their health does not prevent them from going to work, they do not consider themselves as ill. Esin ${ }^{14)}$ in her study on Turkish workers established that as the importance placed on health increased so did healthy life style behaviors.

In this study, the subjects got low scores of exercise, concurrent with the literature ${ }^{9,11,12,14,15)}$. In western countries exercise was considered as jogging, weight lifting or swimming, while in developing countries where life-styles and culture differ from those in the western countries going to school or work on foot, doing housework and job required activities are considered as exercise ${ }^{5)}$. In addition, the unemployed have also been observed to get lower scores on exercise ${ }^{5,23)}$.

In the present study, employees got higher scores on nutrition than on exercise (Table 1), consistent with the literature ${ }^{9,11,12,14,15)}$. Actually, nutrition and exercise are the two important behaviors which play an important role in health. Acquisition of both behaviors is affected by not only perception of health but also the cultural environment where an individual is born and lives. Therefore, occupational health nurses should be aware of health behaviors of employees they provide care for and the factors which shape the behaviors; thus, their attempts to promote health behaviors will be more efficient.

In this study, workers got the highest scores on 
Table 4. Features of workers and their HPLP scores

\begin{tabular}{|c|c|c|c|c|c|c|c|c|}
\hline & & $\begin{array}{c}\text { Self- } \\
\text { actualization }\end{array}$ & $\begin{array}{c}\text { Health } \\
\text { Responsibility }\end{array}$ & Exercise & Nutrition & $\begin{array}{c}\text { Interpersonal } \\
\text { Support }\end{array}$ & $\begin{array}{c}\text { Stress } \\
\text { Management }\end{array}$ & Total \\
\hline \multicolumn{9}{|c|}{$\begin{array}{l}\text { Perceived Health } \\
\text { Status }\end{array}$} \\
\hline Excellent & & $\begin{array}{c}2.93 \\
(0.51)\end{array}$ & $\begin{array}{c}2.52 \\
(0.54)\end{array}$ & $\begin{array}{c}2.33 \\
(0.72)\end{array}$ & $\begin{array}{c}2.59 \\
(0.45)\end{array}$ & $\begin{array}{c}2.92 \\
(0.49)\end{array}$ & $\begin{array}{c}2.62 \\
(0.46)\end{array}$ & $\begin{array}{c}2.66 \\
(0.40)\end{array}$ \\
\hline Well & & $\begin{array}{c}2.72 \\
(0.50)\end{array}$ & $\begin{array}{c}2.26 \\
(0.52)\end{array}$ & $\begin{array}{c}1.96 \\
(0.60)\end{array}$ & $\begin{array}{c}2.37 \\
(0.46)\end{array}$ & $\begin{array}{c}2.77 \\
(0.45)\end{array}$ & $\begin{array}{c}2.35 \\
(0.53)\end{array}$ & $\begin{array}{c}2.41 \\
(0.40)\end{array}$ \\
\hline Poor & & $\begin{array}{c}2.27 \\
(0.41)\end{array}$ & $\begin{array}{c}1.96 \\
(0.52)\end{array}$ & $\begin{array}{c}1.68 \\
(0.38)\end{array}$ & $\begin{array}{c}2.20 \\
(0.44)\end{array}$ & $\begin{array}{c}2.41 \\
(0.54)\end{array}$ & $\begin{array}{c}1.96 \\
(0.44)\end{array}$ & $\begin{array}{c}2.09 \\
(0.37)\end{array}$ \\
\hline & $\mathrm{F}$ & 8.892 & 7.612 & 8.818 & 6.050 & 5.863 & 9.125 & 12.382 \\
\hline & $p$ & $0.000 * * *$ & $0.000 * * *$ & $0.000 * * *$ & $0.003 * *$ & $0.003^{* *}$ & $0.000 * * *$ & $0.000 * * *$ \\
\hline \multicolumn{9}{|l|}{$\begin{array}{l}\text { The important } \\
\text { of health }\end{array}$} \\
\hline None & & $\begin{array}{c}2.11 \\
(0.47)\end{array}$ & $\begin{array}{c}1.62 \\
(0.41)\end{array}$ & $\begin{array}{c}1.25 \\
(0.43)\end{array}$ & $\begin{array}{c}1.93 \\
(0.64)\end{array}$ & $\begin{array}{c}2.33 \\
(0.50)\end{array}$ & $\begin{array}{c}1.82 \\
(0.66)\end{array}$ & $\begin{array}{c}1.85 \\
(0.39)\end{array}$ \\
\hline Little & & $\begin{array}{c}2.59 \\
(0.52)\end{array}$ & $\begin{array}{c}2.13 \\
(0.41)\end{array}$ & $\begin{array}{c}1.93 \\
(0.48)\end{array}$ & $\begin{array}{c}2.24 \\
(0.41)\end{array}$ & $\begin{array}{c}2.61 \\
(0.41)\end{array}$ & $\begin{array}{c}2.15 \\
(0.49)\end{array}$ & $\begin{array}{c}2.28 \\
(0.34)\end{array}$ \\
\hline Middle & & $\begin{array}{c}2.66 \\
(0.51)\end{array}$ & $\begin{array}{c}2.18 \\
(0.49)\end{array}$ & $\begin{array}{c}1.87 \\
(0.57)\end{array}$ & $\begin{array}{c}2.33 \\
(0.46)\end{array}$ & $\begin{array}{c}2.74 \\
(0.48)\end{array}$ & $\begin{array}{c}2.32 \\
(0.53)\end{array}$ & $\begin{array}{c}2.36 \\
(0.39)\end{array}$ \\
\hline Very & & $\begin{array}{c}2.90 \\
(0.45)\end{array}$ & $\begin{array}{c}2.49 \\
(0.49)\end{array}$ & $\begin{array}{c}2.24 \\
(0.63)\end{array}$ & $\begin{array}{c}2.53 \\
(0.42)\end{array}$ & $\begin{array}{c}2.87 \\
(0.44)\end{array}$ & $\begin{array}{c}2.53 \\
(0.47)\end{array}$ & $\begin{array}{c}2.60 \\
(0.37)\end{array}$ \\
\hline \multirow[t]{3}{*}{ Very much } & & $\begin{array}{c}2.99 \\
(0.45)\end{array}$ & $\begin{array}{c}2.59 \\
(0.70)\end{array}$ & $\begin{array}{c}2.36 \\
(0.79)\end{array}$ & $\begin{array}{c}2.65 \\
(0.44)\end{array}$ & $\begin{array}{c}2.97 \\
(0.46)\end{array}$ & $\begin{array}{c}2.65 \\
(0.49)\end{array}$ & $\begin{array}{c}2.71 \\
(0.41)\end{array}$ \\
\hline & $\mathrm{F}$ & 7.251 & 9.509 & 8.744 & 6.800 & 4.016 & 6.580 & 11.909 \\
\hline & $p$ & $0.000 * * *$ & $0.000 * * *$ & $0.000 * * *$ & 0.000 *** & $0.004 * *$ & $0.000 * * *$ & $0.000 * * *$ \\
\hline
\end{tabular}

${ }^{*} p<0.05 ; * * p<0.01 ; * * * p<0.001$.

interpersonal support, consistent with the results of the study by Tokgöz ${ }^{15}$. The results may have been affected by Turkish culture. Indeed, the hallmark of Turkish culture is that family members give support each other during difficult times. Although interpersonal support has no direct influence on health promoting life style, it may help individuals to think and make a move ${ }^{21}$. Therefore, occupational health nurses may encourage individuals to strengthen existing social support and acquire new social support and thus contribute to general well-being of individuals.

As to stress management, the workers got moderate scores, consistent with those reported by $\mathrm{Esin}^{14}$. However, they got lower scores of stress management than reported from other studies $^{2,9,12,15)}$. The low scores of stress management may be explained by working conditions and inability to develop coping strategies. Ahijevych ${ }^{6}$ also found working women to have low stress management scores. Working people have a number of stress related health problems. For this reason, occupational health nurses should be aware of work stress and its causes, make attempts to reduce stress in cooperation with the administration and other health professionals and thus they will protect and promote workers' health.

Demographic features have been found to affect health promotion and life style ${ }^{5,6,8)}$. In fact, age, gender, education level, marital status and perception of health play an important role in health promoting behaviors.

In this study, there was a statistically significant difference between age and nutrition and exercise. But there was no difference between HPLP and subscale scores and education, marital status and gender (Table 2).

Health promoting behaviors have been shown to be positively related to age and education level ${ }^{2,9,21,24)}$. However, Weitzel ${ }^{11)}$ found age to be negatively related to exercise and positively related to health responsibility and nutrition. Bagwell $^{16)}$ found no significant relation between age and total HPLP scores. Tokgöz ${ }^{15)}$ could not find a significant difference between age and total HPLP scores or scores of any subscales. Lusk ${ }^{12}$ reported that there was no significant difference between marital status and HPLP in general and its subscales. In this study, the reason for lack of a relation between age and health behaviors may be that the workers were nearly in the same age range. The finding that education 
has no impact on health behaviors among Turkish workers may be explained by cultural characteristics.

We found a statistically significant difference between income and HPLP scores (Table 2). The workers with an income higher than their expenses received higher HPLP scores, consistent with the results of a study by $\operatorname{Esin}^{14)}$, but conflicting with the results of a study by Pender. Although income alone is not thought to predict health behaviors, it may have a negative effect on health in countries with a low income per capita such as Turkey.

The workers who were positive about their health had a healthier life style. In fact, total HPLP scores and subscale scores revealed that there was a significant difference between perception of health and importance of health. Angela and K1lıan ${ }^{18)}$ in their study on 120 health professionals established a strong positive relation between perception of health and healthy life style behaviors, consistent with the results reported from the other studies ${ }^{2,11,17)}$. In view of the results of this study and other studies, we can conclude that individuals who perceived their health to be very good participate more in health promoting behaviors. Perception of health and illness has an important effect on health behaviors of individuals in Turkey. It is essential that occupational health nurses should know perception and importance of health among workers so that they succeed in their attempts to improve health at workplaces.

\section{Conclusion}

Health promoting lifestyle profile score of Turkish workers are moderate. The workers received the lowest mean scores on exercise and health responsibility. Being healthy and working in healthy environments are two basic human rights. Occupational health nurses have the ability to influence workers' well-being and health. One of the most important responsibilities of occupational health nurses is to protect and promote workers' health. Therefore, they should determine health behaviors of employees and develop health promoting programs when the need arises. The design of workplace and health promotion programs must be based on knowledge of factors influencing the adoption of healthy lifestyles by employees. Employee involvement in planning lifestyle modifications as well as supportive changes in the work environment should also be taken into consideration.

\section{References}

1) Social Insurance Institution (SSK). Number of Work Places and Insured Persons (2003). http://www.ssk.gov.tr/wps/
sskroot/istatistikk/istatistik2003/T1_15.xls. Accessed June 17, 2005.

2) Pender NJ, Walker SN, Sechrist KR, Stromborg MF (1990) Predicting health-promoting lifestyles in the workplace. Nursing Res 39, 326-32.

3) Baltaş Z (2000) Health psychology behavioral sciences and public health (Sağlık ve hastalık anlayışları. Sağlık psikolojisi), Remzi Publishing Company (Remzi Kitabevi), Istanbul.

4) Pender N, Barkauskas V, Hayman L, Rise VH, Anderson E (1992) Health promotion and disease prevention toward excellence in nursing practice and education. Nursing Outlook 40, 106-12.

5) Al Ma'aitah R, Haddad (1999) Health promotion behaviors of Jordanian women. Health Care for Women International 20, 533-47.

6) Ahijevych K, Bernhard L (1994) Health promoting behaviors of African-American women. Nursing Research 42, 86-9.

7) Esin N (1994) Evaluation of Turkish women's health behaviors (Türk kadınlarının sağlık davranişlarının değerlendirilmesi). The Report from the 4th National Public Health Congress (Ulusal Halk Sağlı̆̆ Kongresi Kitabı), 525, Adana.

8) Resnick B (2003) Health promotion practices of older adults: model testing. Public Health Nursing 20, 2-12.

9) Duffy E, Rossow R, Hernandez M (1996) Correlates of health promotion activities in employed Mexican-American women. Nursing Res 45, 18-24.

10) Kuster A, Fong C (1993) Further psychometric evaluation of the Spanish language health promotion lifestyle profile. Nursing Res 42, 266-9.

11) Weitzel MH (1989) A test of the health promotion model with blue collar workers. Nursing Res 38, 99-104.

12) Lusk SL, Kerr MJ, Ronis DV (1995) Health- promoting lifestyles of blue-collar, skilled trade, and white-collar workers. Nursing Res 44, 20-4.

13) Kerr MJ, Ritchey DA (1990) Health-promoting lifestyles of English-speaking and Spanish-speaking Mexican-American migrant farm workers. Public Health Nurs 7, 80-7.

14) Esin N (1997) Evaluation and promotion of health behaviors of industry workers. Istanbul University Health Science Institute an Unpublished PhD Thesis, Istanbul (in Turkish).

15) Tokgöz E (2002) Evaluation of health behaviors among lecturers and of affecting factors (Kadın öğretim elemanlarının sağlığı geliştirme davranışları ve etkileyen etmenlerin değerlendirilmesi). Dokuz Eylul University of Health Sciences Institute, an Unpublished PhD Thesis, Izmir (in Turkish).

16) Bagwell M, Bush $H$ (2000) Improving health promotion for blue-collar workers. J Nursing Quality 14, 65-71.

17) Walker SN, Volkan K, Sechrist KR, Pender NJ (1988) Health promoting life styles of older adults: comparisons with young and middle-aged adults, correlates and patterns. Adv Nurs Sci 11, 76-90.

18) Angela J, Gillis RN (1993) Determinants of a health- 
promoting lifestyle: an integrative review. J Adv Nurs 18, 345-53.

19) Walker SN, Sechrist KR, Pender NJ (1987) Health promotion lifestyle profile development psychometrics. Nursing Res 36, 76-80.

20) Walker SN, Hill-Polerecky DM (1996) Psychometric evaluation of the Health Promoting Lifestyle Profile II, Unpublished manuscript, University of Nebraska Medical Center.

21) Akça Ş (1998) Evaluation of health behaviors among lecturers and of affecting factors, Aegean University Health Science Institute, an Unpublished PhD Thesis, Izmir (in Turkish).

22) Delaney FG (1994) Nursing and health promotion: conceptual concerns. J Adv Nurs 20, 828-35.

23) Chen MY (1999) The effectiveness of health promoting counseling to family caregivers. Public Health Nurs 16, 125-32.

24) Wang HH (1999) Predictors of health promotion lifestyle among three ethnic groups of elderly rural women in Taiwan. Public Health Nurs 16, 321-8. 